\title{
Aortic valve calcification volumes and chronic brain infarctions in patients undergoing transcatheter aortic valve implantation
}

\author{
Wieneke Vlastra ${ }^{1}$-Thomas P. W. van den Boogert ${ }^{1}$ - Thomas Krommenhoek ${ }^{1}$ Anne-Sophie G. T. Bronzwaer ${ }^{2,3}$. \\ Henk J. M. M. Mutsaerts ${ }^{4} \cdot$ Hakim C. Achterberg ${ }^{5}$ - Esther E. Bron ${ }^{5}$. Wiro J. Niessen ${ }^{5}$ Charles B. L. M. Majoie ${ }^{4}$. \\ Aart J. Nederveen ${ }^{4} \cdot$ Jan Baan ${ }^{1} \cdot$ Johannes J. van Lieshout ${ }^{2,3,6}$. Jan J. Piek ${ }^{1} \cdot$ R. Nils Planken ${ }^{4} \cdot$ José P. S. Henriques ${ }^{1}$. \\ Ronak Delewi ${ }^{1}$ (1)
}

Received: 19 April 2019 / Accepted: 1 July 2019 / Published online: 16 July 2019

(c) The Author(s) 2019

\begin{abstract}
Chronic silent brain infarctions, detected as new white matter hyperintensities on magnetic resonance imaging (MRI) following transcatheter aortic valve implantation (TAVI), are associated with long-term cognitive deterioration. This is the first study to investigate to which extent the calcification volume of the native aortic valve (AV) measured with cardiac computed tomography angiography (CTA) predicts the increase in chronic white matter hyperintensity volume after TAVI. A total of 36 patients $\left(79 \pm 5\right.$ years, median EuroSCORE II $\left.1.9 \%, \mathrm{Q}_{1}-\mathrm{Q}_{3} 1.5-3.4 \%\right)$ with severe AV stenosis underwent fluid attenuation inversion recovery (FLAIR) MRI $<24 \mathrm{~h}$ prior to TAVI and at 3 months follow-up for assessment of cerebral white matter hyperintensity volume $(\mathrm{mL})$. Calcification volumes $\left(\mathrm{mm}^{3}\right)$ of the $\mathrm{AV}$, aortic arch, landing zone and left ventricle were measured on the CTA pre-TAVI. The largest calcification volumes were found in the AV (median $692 \mathrm{~mm}^{3}$ ) and aortic arch (median $633 \mathrm{~mm}^{3}$ ), with a large variation between patients $\left(\mathrm{Q}_{1}-\mathrm{Q}_{3} 482-1297 \mathrm{~mm}^{3}\right.$ and $213-1727 \mathrm{~mm}^{3}$, respectively). The white matter hyperintensity volume increased in $72 \%$ of the patients. In these patients the median volume increase was of $1.1 \mathrm{~mL}\left(\mathrm{Q}_{1}-\mathrm{Q}_{3} 0.3-4.6 \mathrm{~mL}\right)$, corresponding with a $27 \%$ increase from baseline $\left(\mathrm{Q}_{1}-\mathrm{Q}_{3} 7-104 \%\right)$. The calcification volume in the AV predicted the increase of white matter hyperintensity volume $(\Delta \%)$, with a $35 \%$ increase of white matter hyperintensity volume, per $100 \mathrm{~mm}^{3}$ of AV calcification volume (SE 8.5, p <0.001). The calcification volumes in the aortic arch, landing zone and left ventricle were not associated with the increase in white matter hyperintensity volume. In $72 \%$ of the patients new chronic white matter hyperintensities developed 3 months after TAVI, with a median increase of $27 \%$. A higher calcification volume in the AV was associated with a larger increase in the white matter hyperintensity volume. These findings show the potential for automated AV calcium screening as an imaging biomarker to predict chronic silent brain infarctions.
\end{abstract}

Keywords Transcatheter aortic valve implantation $\cdot$ Transcatheter aortic valve replacement $\cdot$ Silent brain infarctions $\cdot$ White matter hyperintensities · Cerebral embolizations

Wieneke Vlastra and Thomas P. W. van den Boogert contributed equally as first authors.

Electronic supplementary material The online version of this article (https://doi.org/10.1007/s10554-019-01663-0) contains supplementary material, which is available to authorized users.

Ronak Delewi

r.delewi@amc.nl

Extended author information available on the last page of the article

\section{Background}

Transcatheter aortic valve implantation (TAVI) has been established as an effective treatment of severe aortic valve (AV) stenosis, reducing both clinical symptoms and mortality. Nevertheless, one of the most feared complications, namely the occurrence of stroke, is still observed in $2.4 \%$ of the patients undergoing TAVI [1]. This complication is observed despite the expansion to low-risk populations, the increase in operator experience and the development of improved valve-systems [2]. The cerebral emboli during TAVI are attributed to the dislodgement of aortic arch calcifications during the advancement of the catheter through the orifice and the expansion of 
the implanted valve crushing the calcified native valve [3]. Our previous research showed that stroke after TAVI is associated with poor outcomes, including a sixfold increase of procedural mortality [1]. Nevertheless, clinically overt strokes are only the tip of the iceberg, since shortly after TAVI acute silent cerebral infarctions are visible with diffusion-weighted magnetic resonance imaging (DW-MRI) in up to $78 \%$ of the patients [4]. Unfortunately, currently the majority of the TAVI-induced cerebral emboli remains unpredictable.

Cardiac computed tomography angiography (CTA) scans are part of the routine pre-TAVI work-up and may be used to measure the local calcification burden in the area of the $\mathrm{AV}$ and arch [5]. This is confirmed by a previous study that showed that a large plaque volume in the AV prior to TAVI predicted the occurrence of acute silent brain infarctions several days after TAVI [6]. Despite a high increase of acute silent brain infarctions, these lesions have as yet not been related to long-term cognitive deterioration [7]. Previous research showed that only a proportion of the acute silent brain infarctions, as detected with DW-MRI several days after TAVI, proceeded into chronic loco-regional remnants, which can be identified as white matter hyperintensities on fluid attenuation inversion recovery (FLAIR) MRI sequence, several months after TAVI [7].

In contrast to acute silent brain infarctions, chronic white matter hyperintensities that emerged after the procedural phase were associated with a decrease in cognitive functioning during long-term follow-up [7]. Moreover, in the general elderly population white matter hyperintensities are associated with cognitive impairment, a twofold higher risk of dementia and a threefold higher risk of stroke [8]. TAVI has been developed to improve quality of life, however the increase in TAVI-associated white matter hyperintensities may evoke cognitive deterioration and subsequently compromise self-reliance and increase healthcare costs. If it could be predicted which patients are at risk for developing chronic white matter hyperintensities after TAVI, these high-risk patients could be protected by using additional procedural protective methods, such as cerebral protections devices.

We investigated for the first time to which extent the calcification volume of the native aortic valve, aortic arch, landing zone and left ventricle measured with cardiac CTA, can be used to predict the increase in chronic white matter hyperintensities after TAVI, indicating new chronic brain infarctions.

\section{Methods}

\section{Study population and trial design}

The current study is a single center, prospective trial, initiated and managed by the Heart Center of the Amsterdam
University Medical Center (UMC), a high volume tertiary cardiac care and TAVI center. Patients with severe AV stenosis planned to undergo TAVI of a native valve were eligible for inclusion in the trial. Exclusion criteria for trial participation were known structural brain disease (including a history of stroke or transient ischemic attacks) or presence of an MRI contra-indication. The decision for TAVI treatment was made by our multidisciplinary TAVI team consisting of a cardiac surgeon, an interventional-cardiologist, a radiologist, a geriatric internist and a dedicated nurse-practitioner. Moreover, the dedicated multidisciplinary TAVI-team selected the access route and the valve size, all according to the most recent guidelines [5]. The transfemoral approach was the default access option, alternatively, in patients with severe peripheral artery disease the transaortic route was used. The study protocol was approved by the Institutional Review Boards of the Academic Medical Center and all participants provided written informed consent. The current study is registered at the Netherlands Trial Register (NL7495).

\section{Endpoints}

The main objectives of the study were to determine the distribution of calcification volumes $\left(\mathrm{mm}^{3}\right)$ using CTA in four prespecified sections of the heart and aortic arch of patients undergoing TAVI (Fig. 1) and to determine whether an association exists between the prespecified regional calcification volumes $\left(\mathrm{mm}^{3}\right)$ and the increase in FLAIR-MRI determined cerebral white matter hyperintensity volume ( $\Delta \%$ from baseline $\mathrm{mL}), 3$ months after TAVI. This relative increase $(\Delta \%)$ rather than the actual increase $(\Delta \mathrm{mL})$ was chosen since we expected large variations in baseline white matter hyperintensity volumes between patients and baseline white matter hyperintensity volume is known to predict the actual increase in volume over time $(\Delta \mathrm{mL})$ [9]. Hence, by selecting the relative increase $(\Delta \%)$ we investigated the influence of the TAVI-procedure rather than the baseline patient risk. The secondary objective was to determine clinical (patient) predictors of both the calcification volume $\left(\mathrm{mm}^{3}\right)$ and of actual increase in new white matter hyperintensities $(\mathrm{mL})$.

\section{Cerebral MRI and cardiac CTA acquisition and image analyses}

A detailed description of the image acquisition and analyses is provided as supplementary text with corresponding table and figure (Fig. 1; Supplementary text 1 and Table 1). In brief, the included patients underwent cerebral MRI evaluation of white matter hyperintensities using a FLAIR sequence at a 3-T MRI scanner at baseline $(<24 \mathrm{~h}$ prior to the TAVI procedure) and 3 months after the TAVI. The FLAIR sequence was used because of its sensitivity to measure white matter hyperintensities, appearing as 


\section{Methodology of calcification segmentation}

1) Aortic valve segments

a

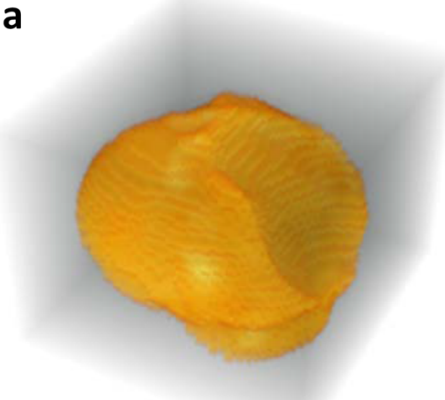

b

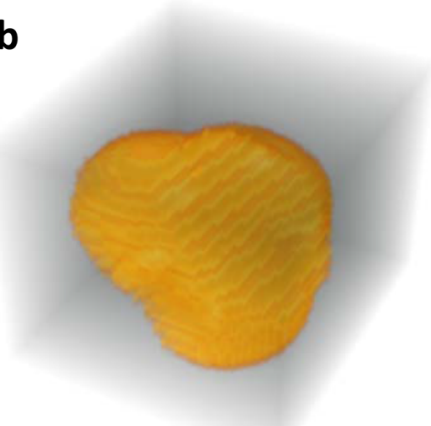

2) Calcification cut-off value determination

a

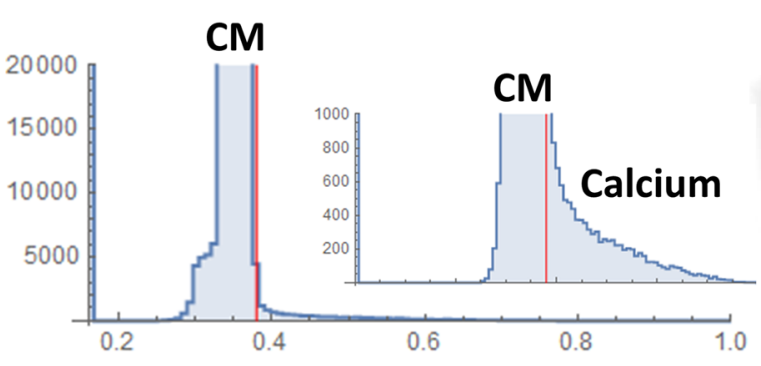

b

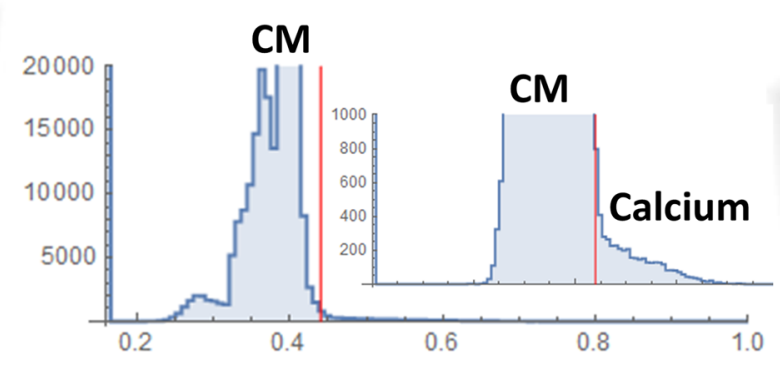

3) Segmented calcification

a

b

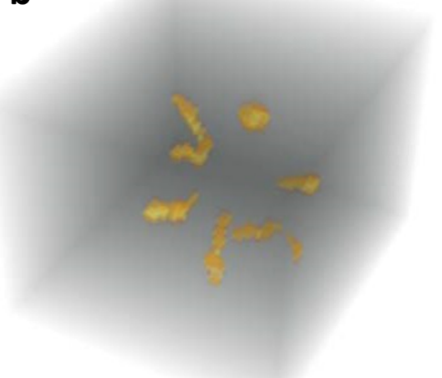

1) Segments of the aortic valve of two patients with either high (a) and low calcification volume (b) are visualized in 3D. 2) A patient specific cut-off value was automatically selected (red lines in the histograms) to create binary masks. 3) Binary masks are used to segment the calcifications from the aortic valve segments in $3 \mathrm{D}$. Note the visual difference in the calcification volume between the patients $a$ and $b$.

Fig. 1 Methodology of calcification segmentation

lesions with increased brightness. White matter hyperintensities were quantified using an automated segmentation method. CTA scans were acquired in all patients as part of the routine pre-TAVI work-up and used to measure the calcification volumes in the selected segments (Fig. 2). In these CTA scans, patient-specific cut-off values for calcifications were automatically detected to minimize differences in calcification volume measurements between patients with different CTA setting [tube voltage $(\mathrm{kV})$ ] and different contrast delivery protocol. These cut-off values were based on the distribution of the amount of pixels with pixel-values, corresponding with the primary components of the segments (calcifications, contrast material and softtissue) (Fig. 1).

\section{Statistical analysis}

Continuous values of patient and MRI characteristics, including calcification volumes per segment and white matter hyperintensity volumes were tested for normal distribution and are reported as mean $\pm \mathrm{SD}$ or median with interquartile ranges $\left(\mathrm{Q}_{1}-\mathrm{Q}_{3} 25-75\right.$ th percentile) where appropriate. Accordingly, depending on the distribution of the data, either the independent t-test or Mann-Whitney $\mathrm{U}$ test determined differences between groups. Categorical variables are presented as frequencies and percentages, differences between groups were tested with Chi-square. Moreover, the calcification volumes per segment $\left(\mathrm{mm}^{3}\right)$ were explored as predictors of the relative increase of white 
Table 1 Baseline patient and procedural characteristics
Study population $(\mathrm{n}=36)$

\begin{tabular}{|c|c|}
\hline \multicolumn{2}{|l|}{ Demographics } \\
\hline Age (years) & $78.7 \pm 4.5$ \\
\hline Female gender & $22(61 \%)$ \\
\hline Body mass index $\left(\mathrm{kg} / \mathrm{m}^{2}\right)$ & $29.8 \pm 7.4$ \\
\hline \multicolumn{2}{|l|}{ Medical history } \\
\hline Previous myocardial infarction & $6(17 \%)$ \\
\hline Previous PCI & $10(28 \%)$ \\
\hline Previous CABG & $3(8 \%)$ \\
\hline Diabetes mellitus & $10(28 \%)$ \\
\hline Hypertension & $21(58 \%)$ \\
\hline Dyslipidemia & $6(17 \%)$ \\
\hline History of coronary artery disease & $14(39 \%)$ \\
\hline Atrial fibrillation & $16(44 \%)$ \\
\hline Glomerular filtration rate $<30 \mathrm{~mL} / \mathrm{min} / 1.73 \mathrm{~m}^{2}$ & $3(8 \%)$ \\
\hline NT-proBNP (ng/L) & $882(361-2775)$ \\
\hline New York Heart Association (NYHA) class III or IV & $22(61 \%)$ \\
\hline \multicolumn{2}{|l|}{ Risk scores } \\
\hline EuroSCORE II (\%) & $1.9(1.5-3.4)$ \\
\hline STS-PROM mortality (\%) & $2.8(1.9-3.7)$ \\
\hline \multicolumn{2}{|l|}{ Echocardiographic characteristics } \\
\hline Aortic max gradient $(\mathrm{mmHg})$ & $68 \pm 24$ \\
\hline Aortic mean gradient (mmHg) & $43 \pm 17$ \\
\hline Aortic valve area $\left(\mathrm{cm}^{2}\right)$ & $0.74 \pm 0.16$ \\
\hline \multicolumn{2}{|l|}{ Procedural details } \\
\hline Transfemoral access & $33(92 \%)$ \\
\hline Transaortic access & $3(8 \%)$ \\
\hline Edwards SAPIEN 3 & $35(97 \%)$ \\
\hline Direct flow & $1(3 \%)$ \\
\hline \multicolumn{2}{|l|}{ Medication at discharge } \\
\hline Single antiplatelet therapy & $1(3 \%)$ \\
\hline Dual antiplatelet therapy & $18(50 \%)$ \\
\hline Single antiplatelet therapy + anticoagulation therapy (VKA/NOAC) & $12(33 \%)$ \\
\hline Anticoagulation therapy only (VKA/NOAC) & $5(14 \%)$ \\
\hline Statin & $24(67 \%)$ \\
\hline
\end{tabular}

Values are mean $( \pm \mathrm{SD}), \mathrm{n}(\%)$ or median (interquartile range)

$V K A$ vitamin $\mathrm{K}$ antagonist, NOAC novel oral anticoagulant matter hyperintensities ( $\Delta \%)$ using linear regression. In a secondary analysis, to correct for inter-individual variance in intracranial volumes, the white matter hyperintensity volume $(\mathrm{mL})$ was normalized for intracranial volume $(\mathrm{mL})$. Moreover, baseline patient characteristics were explored by linear regression as predictors of either the baseline calcification volume per segment $\left(\mathrm{mm}^{3}\right)$ or the increase of white matter hyperintensities $(\Delta \mathrm{mL})$. Potential clinical predictors were tested in a univariate model, and if $\mathrm{p}<0.05$, simultaneously entered to create a multiple regression model. The results of all regression models are reported as beta coefficients (B) and standard errors (SEs). All statistical tests were twotailed, and a value of $p<0.05$ was considered to indicate a statistically significant difference. Calculations were generated using SPSS software (version 25.0 for Windows, SPSS, Inc., Chicago, Illinois).

\section{Results}

\section{Baseline patient characteristics}

Between June 2016 and November 2017, a total of 48 patients with severe AV stenosis in the work-up for TAVI underwent baseline assessment of white matter hyperintensity volume. Twelve patients (25\%) did not undergo 


\section{Segments of the heart and aortic arch used for calcification volume determination}

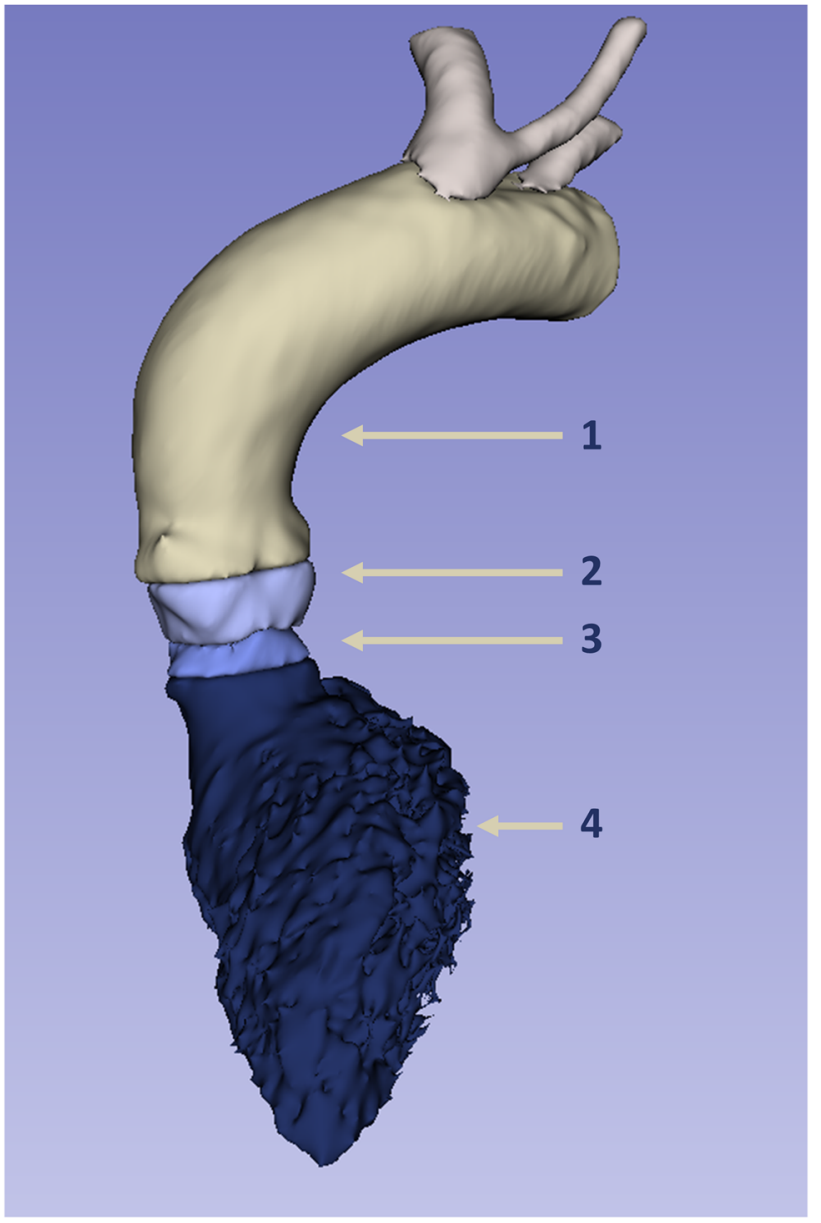

1) aortic arch, starting at the offspring of the left subclavian artery, ending at the distal level of the coronary ostia; 2) aortic valve; 3 ) landing zone, starting immediately below the level of the aortic valve leaflets and including the larger part of the left ventricular outflow tract. This section includes all calcifications adherent to but below the level of the aortic valve; 4) left ventricle, starting below the landing zone and including the mitral valve.

Fig. 2 Segments of the heart and aortic arch used for calcification volume determination

follow-up cerebral MRI 3 months after the procedure related to either post-TAVI pacemaker implantation $(n=6)$ or refusal of MRI follow-up $(n=6)$. Accordingly, the final study population included 36 TAVI patients with complete MRI follow-up. Baseline demographic and clinical characteristics of the patients are provided in Table 1. Mean age was $78.7 \pm 4.5$ years with the majority $(61 \%)$ female. The Society of Thoracic Surgeons (STS) median score of $2.8 \%\left(\mathrm{Q}_{1}-\mathrm{Q}_{3} 1.9-3.7 \%\right)$ indicated this was a low-risk TAVI population. Nevertheless, many patients had comorbidities including diabetes (28\%), atrial fibrillation (44\%) and a history of coronary artery disease (39\%). The majority of the patients underwent transfemoral TAVI $(92 \%)$ with a balloon-expandable Edwards SAPIEN 3 valve (97\%). Following TAVI, $50 \%$ of the patients were treated with dual antiplatelet therapy (DAPT) and 33\% received single antiplatelet therapy (SAPT) in combination with anticoagulant therapy. Patients not undergoing MRI follow-up had a higher median EuroSCORE II (3.4\%, $\mathrm{Q}_{1}-\mathrm{Q}_{3} 2.0-4.6 \%$, vs. $\left.1.9 \%, \mathrm{Q}_{1}-\mathrm{Q}_{3} 1.5-3.5 \%, \mathrm{p}=0.03\right)$ and a larger $\mathrm{AV}$ area $\left(0.91 \pm 0.18 \mathrm{~cm}^{2}\right.$ vs. $0.74 \pm 0.16 \mathrm{~cm}^{2}$ vs., $\left.\mathrm{p}=0.004\right)$ prior to TAVI:(Supplementary Table 2).

\section{Cardiac and aortic calcification volumes}

Out of the four prespecified segments median calcification volumes were the highest in the AV $\left(692 \mathrm{~mm}^{3}\right.$, $\left.\mathrm{Q}_{1}-\mathrm{Q}_{3} 482-1297 \mathrm{~mm}^{3}\right)$ and the aortic arch $\left(633 \mathrm{~mm}^{3}, \mathrm{Q}_{1}-\mathrm{Q}_{3}\right.$ $213-1727 \mathrm{~mm}^{3}$ ). The distribution of the calcification volumes in the AV are displayed in Fig. 3. A CTA example of a low versus a high calcification volume of the $\mathrm{AV}$ is shown in Fig. 4. In multiple regression analysis, AV calcification volumes were larger in patients with a larger (CT-determined) annular area, with a $3.8 \mathrm{~mm}^{3}(\mathrm{SE} 1.2, \mathrm{p}=0.004)$ increase of $\mathrm{AV}$ calcification volume per every additional $\mathrm{mm}^{2}$ of annular area (Supplemental Table 3). Moreover, patients with a higher mean gradient had higher AV calcification volumes, with a $15.9 \mathrm{~mm}^{3}$ (SE 4.9, $\left.\mathrm{p}=0.003\right)$ increase per every additional $\mathrm{mmHg}$ mean gradient increase. The majority of patients with severe AV stenosis (82\%) also had some calcifications in the left ventricle. These calcifications were generally located around the mitral valve and had a lower volume than the AV and arch $\left(164 \mathrm{~mm}^{3}, \mathrm{Q}_{1}-\mathrm{Q}_{3} 19-1332 \mathrm{~mm}^{3}\right)$. Finally, $63 \%$ of the patients had calcifications in the landing zone, with a relatively low calcification volume compared with the other segments $\left(16 \mathrm{~mm}^{3}, \mathrm{Q}_{1}-\mathrm{Q}_{3} 0-68 \mathrm{~mm}^{3}\right)$.

\section{Cerebral white matter hyperintensity volume}

All patients with severe AV stenosis had white matter hyperintensities prior to TAVI, with a median volume of $5.1 \mathrm{~mL}\left(\mathrm{Q}_{1}-\mathrm{Q}_{3} 2.1-8.9 \mathrm{~mL}\right)$, this was $0.4 \%$ of the total intracranial volume $\left(\mathrm{Q}_{1}-\mathrm{Q}_{3} \quad 0.2-0.7 \%\right)$. The volume of baseline white matter hyperintensities varied considerably between patients, the distribution is given in Fig. 3. Figure 5 shows two patients with few versus widespread white matter hyperintensities. Patients with versus without pre-existing atrial fibrillation had a more than twofold higher baseline white matter hyperintensity volume (8.6 mL, $\mathrm{Q}_{1}-\mathrm{Q}_{3} 4.2-13.9 \mathrm{~mL}$ vs. $3.7 \mathrm{~mL}, \mathrm{Q}_{1}-\mathrm{Q}_{3}$ 1.4-6.0 mL, p =0.03). None of the patients experienced clinical overt stroke during the follow-up period. Nevertheless, in 26 patients (72\%) the white matter hyperintensity volume increased 3 months after TAVI. The median 
Fig. 3 Distribution of aortic valve calcification volumes and white matter hyperintensities in patients with severe aortic valve stenosis

\section{Distribution of aortic valve calcification volumes in patients with severe aortic valve stenosis}

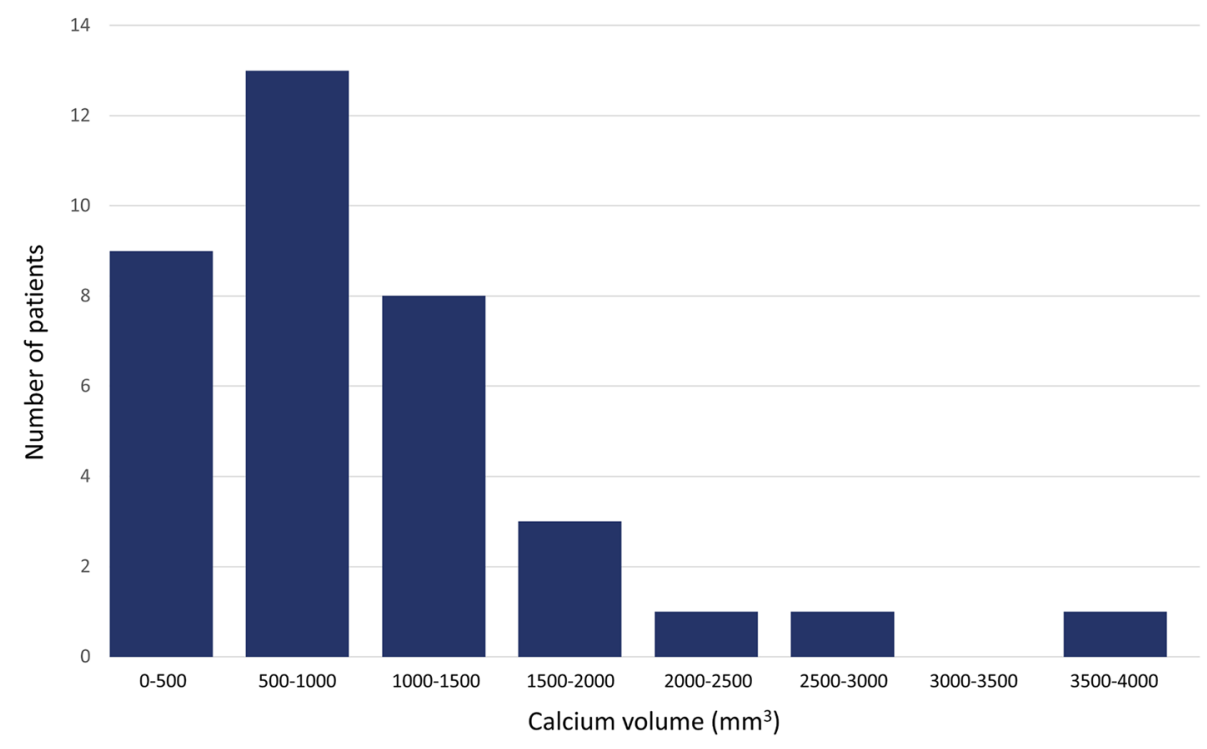

\section{Distribution of white matter hyperintensies in patients with severe aortic valve stenosis}

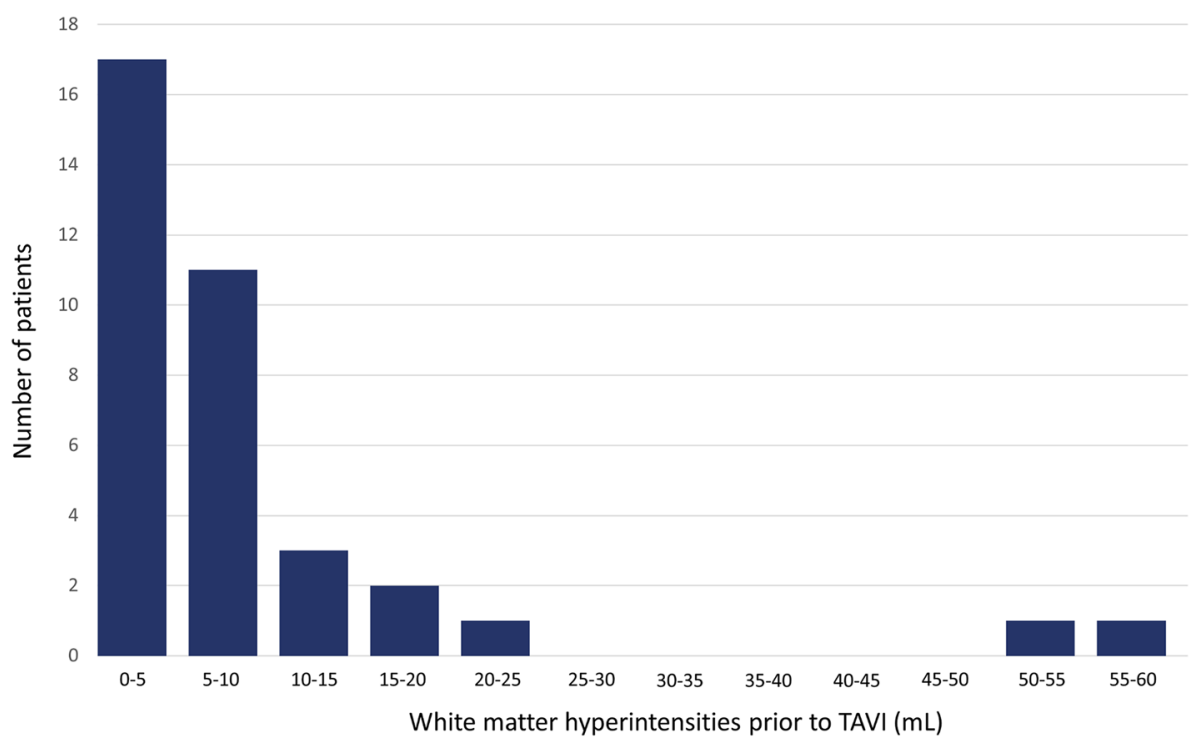

increase in these patients was $1.1 \mathrm{~mL}\left(\mathrm{Q}_{1}-\mathrm{Q}_{3} 0.3-4.6 \mathrm{~mL}\right)$, corresponding with a $27 \%$ increase from baseline $\left(\mathrm{Q}_{1}-\mathrm{Q}_{3}\right.$ $7-104 \%$ ), the distribution of the increase in white matter hyperintensity volume is shown in Fig. 6. The calcification volume of the AV predicted the relative increase of white matter hyperintensity volume at follow-up $(\Delta \%)$, with a $35.2 \%$ increase per $100 \mathrm{~mm}^{3}$ of native AV calcification volume (SE 8.5, $\mathrm{p}<0.001)$. The relationship between AV calcification volume and the increase in white matter hyperintensities is displayed in Fig. 6. In contrast, the calcification volume of the AV was not associated with the volume of baseline white matter hyperintensities (B 0.0, SE $0.0, p=0.87)$. Furthermore, the calcification volume in the aortic arch (B 3.0\%, SE 3.7, p=0.42), landing zone $(\mathrm{B}-31.9 \%$, SE 79.5, $\mathrm{p}=0.69)$ and left ventricle $(\mathrm{B}-3.9 \%$, SE $6.5, p=0.55)$ did not predict the relative increase in cerebral white matter hyperintensity volume. There was a considerable variation in intracranial volumes between patients, with a median volume of $1352 \mathrm{~mL}\left(\mathrm{Q}_{1}-\mathrm{Q}_{3}\right.$ $1205-1468 \mathrm{~mL})$ at baseline. Correction for intracranial 
Cardiac Computed Tomography Angiography (CTA)

Aortic valve calcification volumes

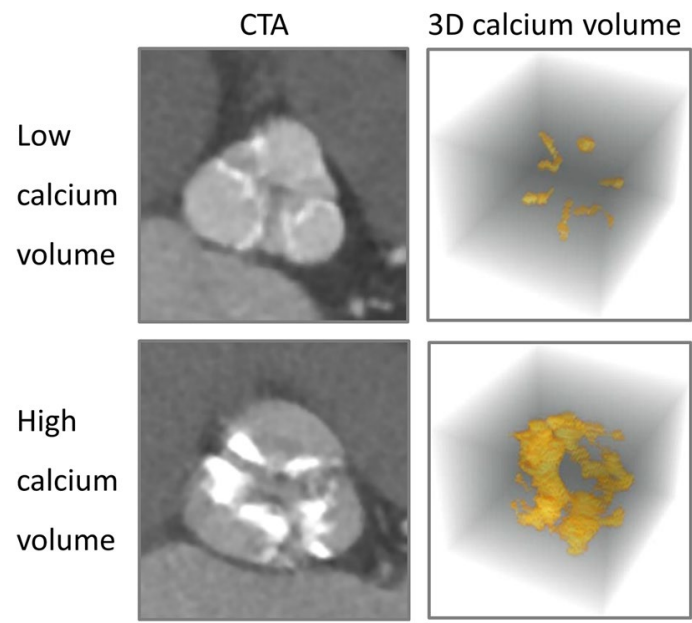

Fig. 4 Cardiac computed tomography angiography (CTA) aortic valve calcification volumes

volumes resulted in similar outcomes, with the AV calcification volume remaining a significant predictor of the relative increase in white matter hyperintensity volume $(\mathrm{p}<0.001)$. In multiple regression analysis, no clinical factors other than the calcification burden of the AV predicted the increase of white matter hyperintensity volume (Supplemental Table 4).

\section{Discussion}

\section{Main findings}

This is the first study to address the relationship between calcification volumes of the heart and aortic arch prior to TAVI and chronic brain infarctions following TAVI. The main findings were that in patients with severe AV stenosis a large variation exists in CTA assessed calcification volumes of the AV. Likewise, the variation in baseline white matter hyperintensity volumes is considerable, with a twofold higher volume in patients with history of atrial fibrillation. Cerebral white matter hyperintensity volume increased in the majority of patients (72\%) during follow-up. A higher volume of AV calcification volume pre-TAVI was associated with a larger increase of white matter hyperintensity volume during follow-up, indicating chronic brain infarctions. Altogether, these findings show the potential for automated $\mathrm{AV}$ calcium screening as an imaging biomarker to predict chronic silent brain infarctions in patients undergoing TAVI.

\section{Whiter matter hyperintensities after TAVI}

The findings of the current study emphasize that the TAVI procedure induces a chronic cerebral embolic burden in an already frail population. In the current low-risk TAVI population, 7 out of 10 patients undergoing TAVI had an increase in white matter hyperintensity volume, with
Fig. 5 Fluid-attenuated inversion recovery (FLAIR) cerebral white matter hyperintensities

\section{Fluid-Attenuated Inversion Recovery (FLAIR)}

\section{Cerebral white matter hyperintensities}
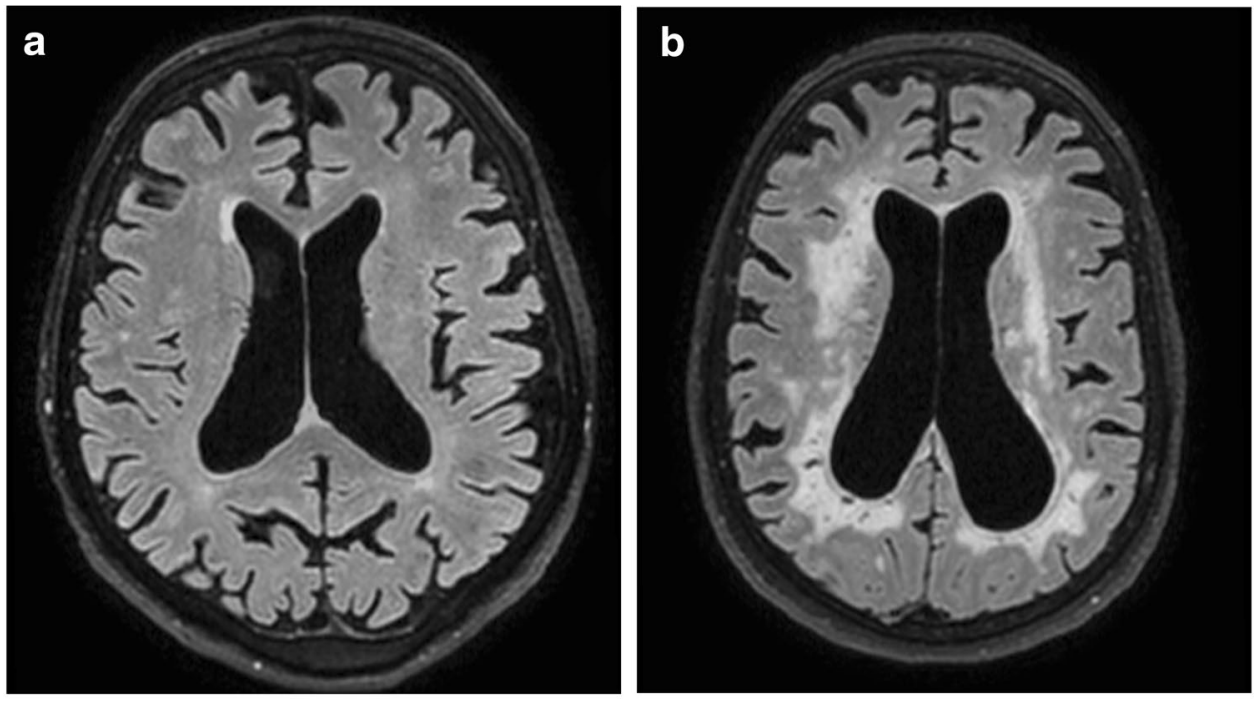

A) Patient with few white matter hyperintensities (Fazekas 1)

B) Patient with widespread white matter hyperintensities (Fazekas 3) 
Fig. 6 Cubic spline curve of the relationship between aortic valve calcification and increase in cerebral white matter hyperintensities. Distribution of the increase in cerebral white matter hyperintensities 3 months after TAVI

\section{Cubic spline curve of the relationship between aortic valve calcification and increase in cerebral white matter hyperintensities}

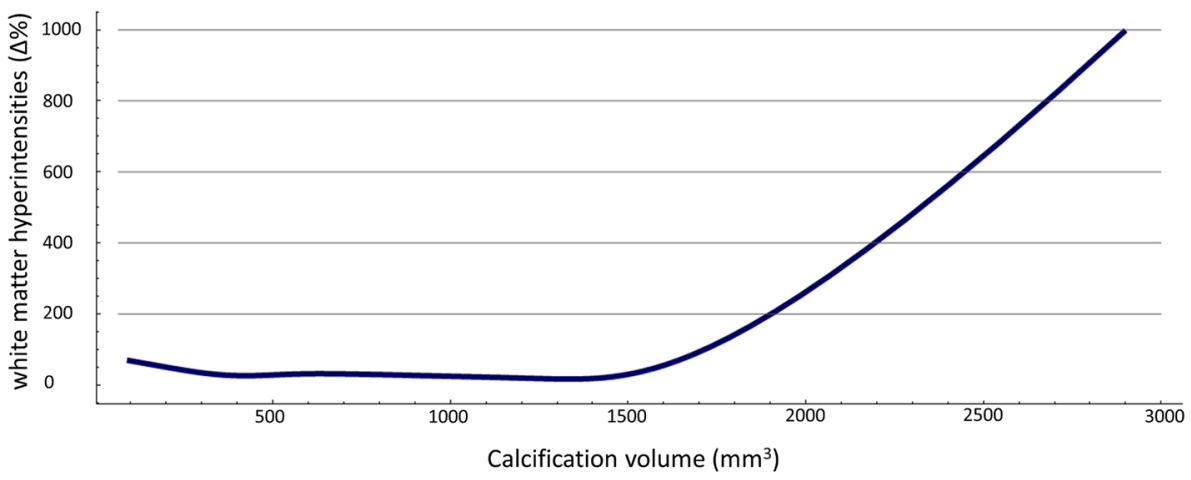

\section{Distribution of the increase in cerebral white matter hyperintensies} 3 months after TAVI

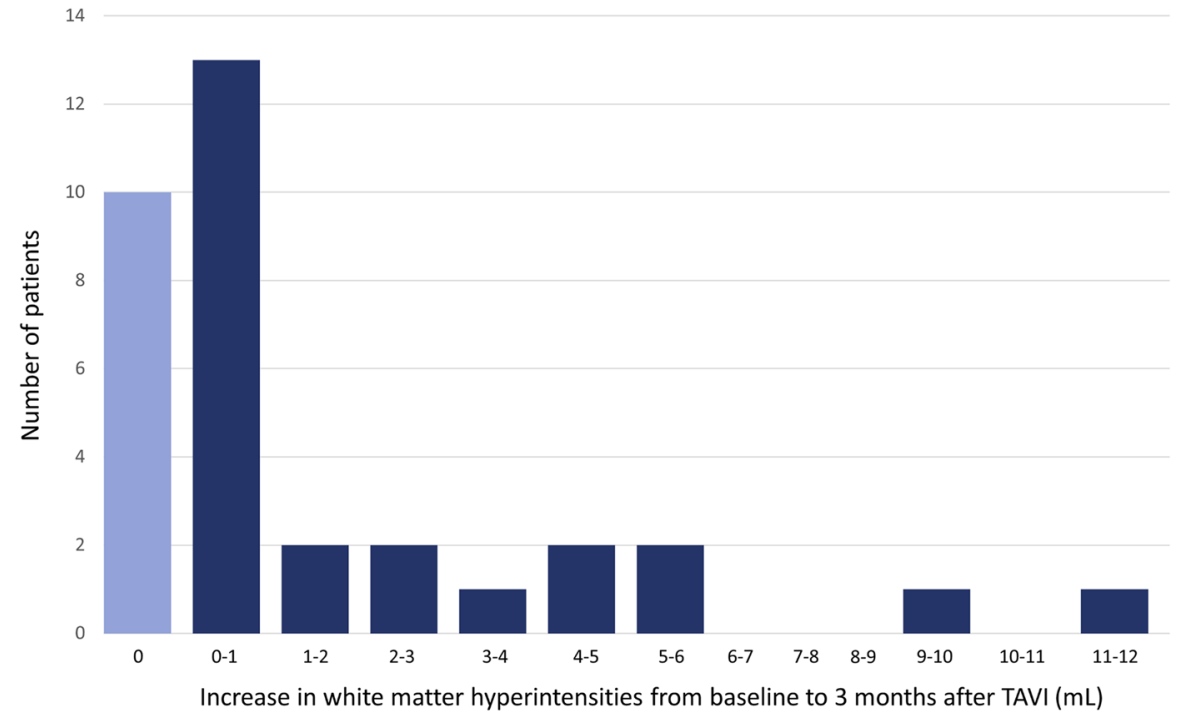

a median increase of $1.1 \mathrm{~mL}\left(\mathrm{Q}_{1}-\mathrm{Q}_{3} 0.3-4.6 \mathrm{~mL}\right)$ in the course of 3 months. In comparison, the mean white matter hyperintensity volume increased with the same volume $(1.1 \pm 1.8 \mathrm{~mL})$ in the course of 4 years in a population-based study in healthy subjects with a comparable mean age of 79 years [10]. Further emphasizing the embolic burden in a portion of the patients, we found that half of the patients had an increase of new white matter hyperintensities $<1 \mathrm{~mL}$, whereas the other half had an increase of 2-12 mL. In population based studies, white matter hyperintensities were related to impaired neuropsychological functioning across all domains including memory, verbal fluency and mental slowing [11].

Similar to findings of the current study, a previous study found that the calcification volume of the AV, measured with the Agatston calcium score, predicted the number of solid cerebral emboli during the TAVI procedure measured with transcranial Doppler [12]. The highest number of cerebral embolizations takes place during the phase of the valve positioning, indicating that calcifications dislocate during valve placement. Similarly, the current results showed that the AV volume predicted the relative increase in white matter hyperintensity volume, rather than the calcification volume in the aortic arch, landing zone and left ventricle. This is despite the fact that large sized catheters are approached through the aortic arch with substantial calcification volumes in almost all patients. This significant role of AV calcifications in cerebral embolizations during the TAVI procedure is underlined by clinical studies indicating that patients with a smaller AV area, higher pre-TAVI AV gradient, increased aortic arch atheroma and/or severe aortic calcification, all indicating a higher calcification burden, are more likely to experience early post-procedural cerebrovascular events [1, 13-16]. Moreover, not the calcification volume itself, but the 
plaque volume in the $\mathrm{AV}$ of a lower density than calcifications (50-130 HU), matching fibrous tissue, was shown to be associated with the occurrence of new DW-MRI lesions short after TAVI [6]. However, studies in TAVI populations did not find a relationship between occurrence of these new DW-MRI lesions after TAVI and (long-term) neurocognitive decline [17]. A mismatch between the presence of acute ischemic lesions detected with DW-MRI and the absence of chronic white matter hyperintensities detected with FLAIR, can be applied to identify salvageable brain areas [18]. In contrast to acute DW-MRI lesions, there is an association between long-term white matter hyperintensities in TAVI patients and a clear trend of cognitive decline, indicating that not all acute silent infarctions continue to be chronic brain infarctions [7]. Accordingly, in contrast to the DWMRI study, the findings of the current study highlight the relationship between calcification volume in patients with $\mathrm{AV}$ stenosis and the increased risk of new white matter hyperintensities after TAVI, which will potentially induce long-term cognitive deterioration.

\section{The risk of atrial fibrillation in TAVI patients}

The reported prevalence of pre-existing atrial fibrillation in TAVI patients ranges from 16 to $51 \%$ [19]. An interesting finding of the current study was that patients with a history of atrial fibrillation had a more than twofold higher volume of baseline white matter hyperintensity volume before TAVI compared without pre-existing atrial fibrillation. This finding is in accordance with data from a large population-based study [20]. Patients with atrial fibrillation are at heightened risk of cognitive decline and dementia, even in the absence of a medical history of past stroke [13]. Moreover, compared with healthy control subjects, patients with atrial fibrillation have a lower cerebral perfusion and impaired cerebrovascular reactivity indicative of a limited cerebral vascular reserve [14]. These findings emphasize the risk of atrial fibrillation on cognitive functioning in patients with AV stenosis.

\section{Reduction of new white matter hyperintensities after TAVI}

Unfortunately, reliable clinical characteristics that predict the risk of (silent) brain infarctions after TAVI are lacking in the existing literature. We believe the findings from the current study, at the background of previous studies, indicate that the routinely performed CTA during pre-TAVI workup could identify patients at risk for TAVI induced chronic silent brain infarctions. Identification of significant ascending aortic atheroma, using preoperative CT, in patients undergoing cardiac surgery, followed by tailored alternative operative strategies led to a threefold lower stroke rate compared with patients who did not undergo aortic atheroma identification with CT $(\mathrm{p}=0.05)$ [15]. In a similar way, the AV calcification volume, measured with automated algorithms in CTA's during the pre-TAVI work-up could assess the risk of cerebral embolization during TAVI and identify patients that could benefit from expensive cerebral protection devices [16].

\section{Limitations}

The current study did not include a control population, that did not undergo TAVI. Moreover, the current study included a low-risk population, whereas the calcification burden may be even higher in intermediate and high-risk populations. Subsequently, a higher calcification burden may be associated with a higher volume of new white matter hyperintensities after TAVI. Similarly, the current study included only balloon-expandable valves, even though self-expandable valves have been associated with a higher stroke rate [21]. Balloon-expandable valves are implanted rather rapidly, in contrast, self-expandable valves are implanted stepwise and slowly and allow repositioning before the final implantation. Therefore, we cannot exclude a relation between the calcification volume in the ascending aorta or left ventricular outflow trajectory and new white matter hyperintensities in patients treated with self-expandable valves. Furthermore, were not able to use the commonly used Agatston calcium score as this has not been validated in contrast enhanced cardiac CTA scans. Non-contrast CT scans of the heart were not part of the TAVI work-up. Moreover, white matter hyperintensities not only include chronic brain infarctions but also regions of small vessel disease. The former is characterized by a rather acute increase of volume, whereas the latter is characterized by a slow gradual increase. Therefore, we believe that by selecting the relative increase $(\Delta \%)$ over the course of a short period of 3 months as the endpoint, the measured outcome mainly detects new infarctions rather than small vessel disease. Finally, cognitive functioning tests were not performed in the current study and relationships between the AV calcification volume, the volume of new white matter hyperintensities and cognitive functioning cannot be confirmed. Even though various studies reported a relationship between the increase in white matter hyperintensities and neuropsychological functioning, there is currently limited evidence reporting an increase in white matter hyperintensity volumes is associated with neurocognitive impairment $[22,23]$.

\section{Conclusions}

In a low-risk population with severe $\mathrm{AV}$ stenosis, all patients had valve calcifications prior to TAVI, with a large variation in calcification volumes between patients. The majority of the 
patients (72\%) had new cerebral white matter hyperintensities 3 months after TAVI, with a median increase of $27 \%$. A larger calcification volume in the $\mathrm{AV}$, but not in the aortic wall, landing zone and left ventricle prior to TAVI was associated with a higher increase in cerebral white matter hyperintensity volume after TAVI, indicating new chronic brain infarctions.

Funding We acknowledge the support from the Netherlands CardioVascular Research Initiative: The Dutch Heart Foundation (CVON 2012-16 Heart Brain Connection), Dutch Federation of University Medical Centres, The Netherlands Organisation for Health Research and Development and The Royal Netherlands Academy of Sciences.

\section{Compliance with ethical standards}

Conflict of interest Dr. Baan receives an unrestricted research Grant from Edwards Lifesciences. The remaining authors have no relevant disclosures.

Ethical approval All procedures were in accordance with the Ethical Standards of the Institutional and/or National Research Committee and with the 1964 Helsinki Declaration and its later amendments or comparable ethical standards.

Informed consent Informed consent was obtained from all individual participants included in the study.

Open Access This article is distributed under the terms of the Creative Commons Attribution 4.0 International License (http://creativeco mmons.org/licenses/by/4.0/), which permits unrestricted use, distribution, and reproduction in any medium, provided you give appropriate credit to the original author(s) and the source, provide a link to the Creative Commons license, and indicate if changes were made.

\section{References}

1. Vlastra W, Jimenez-Quevedo P, Tchetche D, Chandrasekhar J, de Brito Jr FS, Barbanti M et al (2019) Predictors, incidence, and outcomes of patients undergoing transfemoral transcatheter aortic valve implantation complicated by stroke. Circ Cardiovasc Interv 12(3): 0007546

2. Mack MJ, Leon MB, Thourani VH, Makkar R, Kodali SK, Russo $M$ et al (2019) Transcatheter aortic-valve replacement with a balloon-expandable valve in low-risk patients. N Engl J Med 380:1695-1705

3. Vlastra W, Piek JJ, Delewi R (2017) The current status of antiplatelet therapy in patients undergoing transcatheter aortic valve implantation. J Thorac Dis 9(10):3652-3655

4. Pagnesi M, Martino EA, Chiarito M, Mangieri A, Jabbour RJ, Van Mieghem NM et al (2016) Silent cerebral injury after transcatheter aortic valve implantation and the preventive role of embolic protection devices: a systematic review and meta-analysis. Int $\mathbf{J}$ Cardiol 221:97-106

5. Baumgartner H, Falk V, Bax JJ, De Bonis M, Hamm C, Holm PJ et al (2017) 2017 ESC/EACTS Guidelines for the management of valvular heart disease. Eur Heart J 38(36):2739-2791

6. Tada N, Haga Y, Suzuki S, Enta Y, Miyasaka M, Inoue H et al (2017) Computed tomography score of aortic valve tissue may predict cerebral embolism during transcatheter aortic valve implantation. JACC Cardiovasc Imaging 10(8):960-962
7. Ghanem A, Dorner J, Schulze-Hagen L, Muller A, Wilsing M, Sinning JM et al (2017) Subacute subclinical brain infarctions after transcatheter aortic valve implantation negatively impact cognitive function in long-term follow-up. PLoS ONE 12(1):e0168852

8. Debette S, Markus HS (2010) The clinical importance of white matter hyperintensities on brain magnetic resonance imaging: systematic review and meta-analysis. BMJ 341:c3666

9. Schmidt R, Fazekas F, Kapeller P, Schmidt H, Hartung HP (1999) MRI white matter hyperintensities: three-year follow-up of the Austrian Stroke Prevention Study. Neurology 53(1):132-139

10. Whitman GT, Tang Y, Lin A, Baloh RW (2001) A prospective study of cerebral white matter abnormalities in older people with gait dysfunction. Neurology 57(6):990-994

11. de Groot JC, de Leeuw FE, Oudkerk M, Hofman A, Jolles J, Breteler MM (2001) Cerebral white matter lesions and subjective cognitive dysfunction: the Rotterdam Scan Study. Neurology 56(11):1539-1545

12. Aggarwal SK, Delahunty Rn N, Menezes LJ, Perry R, Wong B, Reinthaler M et al (2018) Patterns of solid particle embolization during transcatheter aortic valve implantation and correlation with aortic valve calcification. J Interv Cardiol 31(5):648-654

13. Miyasaka Y, Barnes ME, Petersen RC, Cha SS, Bailey KR, Gersh $B J$ et al (2007) Risk of dementia in stroke-free patients diagnosed with atrial fibrillation: data from a community-based cohort. Eur Heart J 28(16):1962-1967

14. Junejo RT, Braz ID, Lucas SJE, van Lieshout JJ, Lip GYH, Fisher JP (2019) Impaired cerebrovascular reactivity in patients with atrial fibrillation. J Am Coll Cardiol 73(10):1230-1232

15. Lee R, Matsutani N, Polimenakos AC, Levers LC, Lee M, Johnson RG (2007) Preoperative noncontrast chest computed tomography identifies potential aortic emboli. Ann Thorac Surg 84(1):38-41; discussion 2

16. Vlastra W, Vendrik J, Koch KT, Baan J, Piek JJ, Delewi R (2018) Cerebral protection devices during transcatheter aortic valve implantation. Trends Cardiovasc Med 28(6):412-418

17. Ghanem A, Kocurek J, Sinning JM, Wagner M, Becker BV, Vogel $\mathrm{M}$ et al (2013) Cognitive trajectory after transcatheter aortic valve implantation. Circ Cardiovasc Interv 6(6):615-624

18. Thomalla G, Cheng B, Ebinger M, Hao Q, Tourdias T, Wu O et al (2011) DWI-FLAIR mismatch for the identification of patients with acute ischaemic stroke within $4.5 \mathrm{~h}$ of symptom onset (PRE-FLAIR): a multicentre observational study. Lancet Neurol 10(11):978-986

19. Tarantini G, Mojoli M, Urena M, Vahanian A (2017) Atrial fibrillation in patients undergoing transcatheter aortic valve implantation: epidemiology, timing, predictors, and outcome. Eur Heart J 38(17):1285-1293

20. de Leeuw FE, de Groot JC, Oudkerk M, Kors JA, Hofman A, van Gijn J et al (2000) Atrial fibrillation and the risk of cerebral white matter lesions. Neurology 54(9):1795-1801

21. Vlastra W, Chandrasekhar J, Munoz-Garcia AJ, Tchetche D, de Brito Jr FS, Barbanti M et al (2019) Comparison of balloonexpandable vs. self-expandable valves in patients undergoing transfemoral transcatheter aortic valve implantation: from the CENTER-Collaboration. Eur Heart J 40(5):456-465

22. Jokinen H, Kalska H, Ylikoski R, Madureira S, Verdelho A, van der Flier WM et al (2009) Longitudinal cognitive decline in subcortical ischemic vascular disease- the LADIS Study. Cerebrovasc Dis 27(4):384-391

23. Steffens DC, Potter GG, McQuoid DR, MacFall JR, Payne ME, Burke JR et al (2007) Longitudinal magnetic resonance imaging vascular changes, apolipoprotein E genotype, and development of dementia in the neurocognitive outcomes of depression in the elderly study. Am J Geriatr Psychiatry 15(10):839-849 


\section{Affiliations}

Wieneke Vlastra $^{1}$ - Thomas P. W. van den Boogert ${ }^{1}$ - Thomas Krommenhoek ${ }^{1}$ Anne-Sophie G. T. Bronzwaer ${ }^{2,3}$. Henk J. M. M. Mutsaerts ${ }^{4}$ Hakim C. Achterberg ${ }^{5}$. Esther E. Bron ${ }^{5}$. Wiro J. Niessen ${ }^{5}$. Charles B. L. M. Majoie ${ }^{4}$. Aart J. Nederveen ${ }^{4}$. Jan Baan ${ }^{1}$. Johannes J. van Lieshout ${ }^{2,3,6}$. Jan J. Piek ${ }^{1}$ R. Nils Planken ${ }^{4}$. José P. S. Henriques ${ }^{1}$. Ronak Delewi ${ }^{1} \mathbb{0}$

1 Department of Clinical and Experimental Cardiology, Amsterdam Cardiovascular Sciences, Amsterdam UMC, University of Amsterdam, Heart Center, Amsterdam, The Netherlands

2 Department of Internal Medicine, Amsterdam UMC, University of Amsterdam, Amsterdam, The Netherlands

3 Laboratory for Clinical Cardiovascular Physiology, Center for Heart Failure Research, Academic Medical Center, University of Amsterdam, Amsterdam, The Netherlands
4 Department of Radiology and Nuclear Medicine, Amsterdam University Medical Centers, Location AMC and VUmc, University of Amsterdam, Amsterdam, The Netherlands

5 Department of Radiology \& Nuclear Medicine, Biomedical Imaging Group Rotterdam, Erasmus MC-University Medical Center Rotterdam, Rotterdam, The Netherlands

6 MRC/Arthritis Research UK Centre for Musculoskeletal Ageing Research, School of Life Sciences, The Medical School, University of Nottingham Medical School, Queen's Medical Centre, Nottingham, UK 